\title{
Linx
}

Revue des linguistes de l'université Paris X Nanterre

$60 \mid 2009$

Nonne scripta manent?

\section{Remarques sur la ponctuation du discours direct dans les Contes de Perrault et de Mme d'Aulnoy}

Remarks on the use of punctuation in direct speech in Perrault's and Mme d'Aulnoy's Tales

\section{Catherine Boré}

\section{OpenEdition}

\section{Journals}

Édition électronique

URL : http://journals.openedition.org/linx/673

DOI : $10.4000 /$ linx.673

ISSN : 2118-9692

\section{Éditeur}

Presses universitaires de Paris Nanterre

Édition imprimée

Date de publication : 1 mai 2009

Pagination : 47-66

ISSN : 0246-8743

\section{Référence électronique}

Catherine Boré, «Remarques sur la ponctuation du discours direct dans les Contes de Perrault et de Mme d'Aulnoy », Linx [En ligne], 60 | 2009, mis en ligne le 02 avril 2012, consulté le 27 mai 2019. URL : http://journals.openedition.org/linx/673; DOI : 10.4000/linx.673 


\title{
Remarques sur la ponctuation du discours direct dans les Contes de Perrault et de Mme d'Aulnoy
}

\author{
Catberine Boré \\ Université de Cergy (IUFM) - UMR 7114 MoDyCo
}

Le discours direct est particulièrement bien représenté dans les contes, notamment dans ces parangons du genre que sont les Contes de Perrault et de Mme d'Aulnoy, tous deux exactement contemporains. Dans cet article, j'aborde le discours direct (désormais DD) par ses marques extérieures, les signes de ponctuation.

Pour autant, le choix d'un corpus de contes du XVIİ̀me siècle est paradoxal : la ponctuation est rare; les signes modernes signalant le DD (guillemets, tirets, alinéas) manquent tous ; les signes de ponctuation sont beaucoup moins nombreux que dans le système actuel, et leur répartition semble à première vue aléatoire.

D'où les questions :

- Comment le DD est-il signalé dans un corpus ancien?

- Les marques qui le constituent sont-elles à chercher dans les seules oppositions de rupture énonciative entre discours citant et discours cité ?

- Ou bien est-il possible de parler d'un système de ponctuation du DD, même restreint à quelques unités ?

- Quel rôle joue alors cette ponctuation dans la production du sens ?

L'article part du principe que la ponctuation n'est pas un simple ornement du discours, mais qu'elle « contribue à la production du sens en tant qu'organisateur de la séquentialité et indicateur syntagmatique et énonciatif (Anis, 1988, p. 246, pour une définition du signe de ponctuation). En ce sens, une telle définition corrige la première approximation de «marque extérieure » au DD par laquelle j’ai commencé : le signe de ponctuation est en effet non lié morphologiquement au texte qu'il accompagne, mais, 
selon le propos de Dahlet (2005, p. 19) auquel je souscris, « elle [la ponctuation] est consubstantielle à la mise en texte ; elle est elle-même opérateur syntaxique et opérateur sémantique. »

C'est donc, selon l'époque considérée, une conception différente du texte que révèle la ponctuation, une conception différente, aussi, du DD et du rôle de l'auteur et/ou du narrateur, comme responsable de l'énonciation. Telle est en tout cas l'hypothèse que je retiendrai.

\section{Le corpus}

Mes observations s'appuient sur deux corpus principaux ${ }^{1}$ : les Histoire on contes du temps passé de Perrault (8 contes en prose) dont je compare l'édition de 1697 (édition princeps Barbin, Paris) avec celle de 1785 du Cabinet des fées (Amsterdam), toutes deux numérisées ${ }^{2}$ et scrupuleusement restituées ${ }^{3}$ des éditions originales.

Pour une comparaison ponctuelle entre auteurs, j’ai utilisé un corpus numérisé ${ }^{4}$ contemporain de celui de Perrault : celui des Contes de Mme d'Aulnoy (25 Contes avec leur nouvelle-cadre) dans l'édition de 1785 du Cabinet des Fées. Rien n'a été conservé des manuscrits de $\mathrm{M}^{\mathrm{me}}$ d'Aulnoy et seule l'édition des premiers livres de 1697 est consultable sur microfiches à la Bibliothèque Nationale : les éditions originales ${ }^{5}$ de ce corpus manquent donc, irrémédiablement. Les premières rééditions des Contes de Mme d'Aulnoy (celles de 1710, 1725, etc.) sont consultables à la Bibliothèque Nationale et non numérisées. Je disposais aussi d'une édition (non numérisée) de 1757 («tome second Les Contes des Fées, Nouvelle édition à Paris Par la Compagnie des Libraires Avec privilège du roi »), antérieure de 30 ans à celle du Cabinet des Fées : ce point de comparaison le plus proche du Cabinet des Fées permet d'établir que le système de ponctuation du DD à cette époque est encore dans la continuité des éditions du XVII ème siècle. La rupture se fait manifestement sous l'influence des grammairiens (Beauzée notamment, article «Ponctuation» de l'Encyclopédie, t. 13, 1765) car l'édition du Cabinet des Fées diffère notablement des précédentes.

La disproportion de taille entre les deux recueils est telle qu'il est impossible, dans le cadre limité d'un article, de procéder à une comparaison fondée. Aussi, après avoir étudié les différences entre les deux corpus de Perrault (éditions 1697 et 1785 des Contes), me contenterai-je de comparer, sur certains attributs, un extrait d'un seul (et long) conte de Mme d'Aulnoy aux extraits des huit (brefs) contes en prose du recueil de Perrault.

\footnotetext{
${ }^{1}$ Réunis en collaboration avec Christian Bouillon, CRTF, Université de Cergy-Pontoise.

2 Base de données Basile, édition Classiques Garnier numérique, issue de l'édition Champion électronique.

${ }^{3}$ En particulier la ponctuation et l'orthographe originales. Toutefois, j'ai adopté l'orthographe modernisée, également disponible sous forme numérisée, pour citer les extraits.

${ }^{4}$ Également disponible dans la base de données Basile, destinée aux lycées et collèges.

${ }^{5}$ Qui ont paru en 1697 et 1698.
} 


\subsection{Les aménagements $d u$ texte de Perrault}

Quand Perrault publia ses Histoire ou contes du temps passé en 1697, tous les contes étaient imprimés pour la première fois sauf un (La Belle au Bois dormant), déjà paru dans le Mercure Galant en 1696. Le seul exemplaire de manuscrit de Perrault existant à ce jour est le manuscrit de Pierpont ${ }^{6}$ (voir Annexe). Il s'agit d'un manuscrit de 1695, probablement de la main d'un copiste, et qui n'est (sans doute) pas celui qui donna lieu à l'édition de 1697. En effet, le texte de cette dernière diffère sensiblement du manuscrit de 1695, qui, en outre, ne contient que cinq contes. Aucune copie d'un (ou de plusieurs ?) manuscrit(s) intermédiaire(s) n’a semble-t-il survécu, qui permettrait d'expliquer l'apparition des trois derniers contes ainsi que les aménagements importants concernant les cinq autres.

Le manuscrit de 1695 est quasi dénué de ponctuation ${ }^{7}$; celle-ci a été ajoutée au moment de l'édition ainsi que les accents. Le peu de ponctuation qu'il y avait a souvent été altéré en suivant les règles des typographes; l'orthographe et la capitalisation ont été l'objet de grandes transformations.

Quelles que soient les lacunes dans l'élaboration manuscrite du matériau des Contes, les deux éditions contrastées de Barbin (Paris, 1697) et du Cabinet des Fées (Amsterdam, 1785), telles qu'elles nous sont parvenues, possèdent leur logique propre et nous renseignent - c'est l'hypothèse que je fais ici - sur la valeur sémantique et énonciative de la ponctuation du discours direct, devenue graduellement, entre ces deux dates, un objet intéressant la grammaire et le style.

\subsection{Préalable: question(s) de méthode}

Bien qu'il existe depuis plus de dix ans des outils statistiques pour analyser la répartition et la fréquence comparative des signes de ponctuation par genre 8 (surtout dans le roman), je n'y ai pas eu recours directement dans l'étude présentée. Tout juste suis-je partie d'informations qui justifient en elles-mêmes que puisse se poser la question de la ponctuation comme élément majeur de l'interprétation des discours directs dans les œuvres anciennes, représentées ici par les Contes de Perrault et de Mme d'Aulnoy.

Dans un article déjà ancien, Sylvie Durrer (1990, p. 37), justifiant son étude du dialogue romanesque par la place qu'il occupe dans une série représentative de dix romans français publiés entre 1964 et 1978, trouvait des pourcentages de dialogue (entendre: discours direct) allant de 13 à 49\%. La variation est importante et suggèrerait à elle seule une analyse plus précise ${ }^{9}$; mais le point de discussion provient de la note 2 :

\footnotetext{
${ }^{6}$ Reproduit en fac-similé dans Barchilon (1956).

${ }^{7}$ L'étude d'I. Barko (1977) sur les lettres manuscrites de Racine montre que c'était l'usage normal du temps.

${ }^{8}$ Voir par exemple les études respectives d’E. Bourion («Ponctuation et accès sémantique aux banques textuelles») et A. Dister («Problématique des fins de phrase en traitement automatique du français »), in Defays et al. (1998, p. 409-435 et 437-447).

${ }^{9}$ Voir sur ce point les travaux de D. Malrieu sur les romans français, notamment ceux de M. Duras. $C f$. par exemple "Familles narratologiques et balisage du roman contemporain », site Infolang Actes ALLC.pdf.
} 
Les pourcentages ont été obtenus en comptant les lignes occupées par les dialogues au discours direct par rapport au montant total des lignes du roman. Comptait pour une ligne toute réplique quelle que soit sa longueur. Lorsqu'une réplique occupait plus d'une ligne n'étaient comptées comme unités complémentaires que celles qui faisaient au moins la moitié d'une ligne.

Il n'est évidemment pas question de reprocher à l'auteur le caractère quelque peu artisanal de ces comptages, à une époque où les corpus numérisés des œuvres en cause n'existaient pas.

En revanche, il semble aller de soi, dans cette démarche, que les répliques de dialogue au DD soient repérables par des lignes, ce qui sous-entend qu'elles soient distinguées du reste de la narration. Voilà qui semble difficile, quel qu'ait pu être le type de présentation adopté par les éditeurs disposant de tout l'arsenal typographique moderne: guillemets, alinéas, tirets. Pour ne prendre qu'un exemple, l'incise, qui s'insinue parfois très longuement dans le discours qu'elle rapporte et qui le fragmente souvent en débordant sur plusieurs lignes, fera-t-elle, ou non, partie du comptage ? Faudra-t-il compter les tronçons de DD qu'elle rapporte au même titre que la réplique d'un seul mot, isolée par un tiret, dont le verbe introducteur a soigneusement été distingué ?

J'ai essayé, à titre d'exemple, de comparer le mode de comptage de DD par lignes selon les principes de S. Durrer à celui, très rudimentaire d'ailleurs, de la fonction statistique de Word en nombre de mots, comparaison effectuée sur le seul corpus Perrault ${ }^{10}$. Les résultats obtenus diffèrent suffisamment pour que l'on s'interroge : pour ce même corpus, en effet, le comptage par ligne donne $27 \%$ de DD, contre $20,75 \%$ par comptage en nombre de mots.

Si ces deux chiffres mettent bien au jour l'importance quantitative du DD dans les Contes, les différences de résultats me semblent s'expliquer par le fait que le comptage par mots ignore le rôle de la ponctuation tandis que le comptage par lignes semble impliquer avec la ligne un « gabarit » naturel isolant le DD.

Le paradoxe de cet exercice est que, justement, la ponctuation des textes anciens, en cette fin du XVII ème siècle où paraissent à la fois les Contes de Perrault et ceux de Mme d'Aulnoy, est très déficitaire par rapport aux Modernes : comme nous allons le voir pour Perrault, l'édition de 1697 des Contes ne comporte ni tiret ni guillemets ni retour à la ligne, et souvent même pas les deux points pour signaler l'apparition d'un DD. Il en résulte une difficulté réelle pour isoler celui-ci : du même coup, la ligne apparaît tout à fait inopérante pour isoler un DD qui se situe à cheval sur plusieurs lignes enfouies dans la narration, comme ici :

(1) Elle y alla quelques jours aprés, et dit un soir à son Maistre d'Hôtel, je veux manger demain à mon dîner la petite Aurore. Ah! Madame, dit le Maistre d'Hôtel; je le veux, dit la Reine (et elle le dit d'un ton d'Ogresse, qui a envie de manger de la chair fraische) et je la veux manger à la Sausserobert. (La Belle au bois dormant)

La revue en ligne Corpus publie régulièrement des études statistiques d'envergure à partir de corpus numériques littéraires dont le balisage a été problématisé.

${ }^{10}$ Je n'ai pas effectué de balisage : la délimitation est donc manuelle, ce qui reste possible pour un petit corpus comme celui des Contes en prose de Perrault (16.264 mots), mais non pour celui d'Aulnoy. 
En revanche une édition moderne destinée à la jeunesse ${ }^{11}$ donne ceci pour le même texte :

(2) Elle y alla quelques jours après, et dit un soir à son maittre d'hôtel :

- Je veux manger demain à mon dîner la petite Aurore.

- $\quad$ Ah! Madame, dit le maitre d'hôtel...

- Je le veux, dit la Reine (et elle le dit d'un ton d'ogresse, qui a envie de manger de la chair fraîche) et je la veux manger avec oignons et moutarde.

Dans l'édition moderne pour la jeunesse, le comptage par lignes en donnera 4 (ou 3 si l'on hésite à compter pour une ligne la phrase de dialogue suivie d'une parenthèse), tandis que pour l'édition de 1697, on peut hésiter entre 2 et 3 (la première ligne étant majoritairement occupée par la narration), d'où un certain flottement. Ainsi s'explique, dans le corpus Perrault, le résultat plus élevé du comptage par lignes sur le comptage par mots : il me semble un effet de l'absence des frontières typo-visuelles marquées entre discours citant et discours cité, la ligne ne fournissant de cadre approprié pour le repérage des paroles rapportées qu’à condition de « réécrire » la ponctuation.

\section{La ponctuation du DD dans les Contes : définitions}

La première chose qui frappe dans les corpus anciens est la difficulté à trouver une hiérarchie dans le niveau de segmentation qu'induit le signe de ponctuation dans le texte.

Jusqu'au milieu du XVIII ème siècle, les signes employés sont peu nombreux: alinéa, point, point-virgule, virgule, qui ont une force décroissante ${ }^{12}$. Surtout, ce sont des signes syntagmatiques - dans la terminologie d'Anis (1988) - car ils segmentent le texte, séquentiels - dans celle de Dahlet (2005) - car ils regroupent des constituants ; les deux-points, point interrogatif et point admiratif (exclamatif), dont la portée et l'usage sont fluctuants, sont des signes énonciatifs ou polyphoniques (Anis), de même que les parenthèses, car ils présupposent un allocutaire. Ajoutons les capitales et les italiques, signes expressifs dont l'usage au XVIİ̀me siècle est pléthorique pour les premières, restreint à l'usage de citation pour les secondes.

Une caractéristique de la ponctuation du discours direct au XVII ème siècle est donc l'absence des tirets, guillemets et alinéas, ce que plusieurs études antérieures ont souligné en remarquant que cet usage s'étend avec quelques nuances jusque dans la première moitié du XVIIIİme siècle. Sonia Branca-Rosoff ${ }^{13}$, étudiant les marques du discours rapporté dans Manon Lescaut tout au long du XVIIIème siècle, montre comment les imprimeurs usent d'une ponctuation d'abord marquée par l'oralité puis de

\footnotetext{
11 Édition Grasset «Monsieur Chat », 1984. Pour comparer des choses comparables, les textes sont alignés de la même façon par le traitement de texte. Seuls les passages à la ligne avec tirets diffèrent entre les deux éditions, ainsi que la ponctuation expressive des points de suspension.

12 Voir Tilkin, 1998, p. 201 qui constate que ce sont surtout les trois derniers signes qui, encore à l'époque de Voltaire, accompagnent les paroles des personnages, les autres signes étant rarissimes.

13 Branca-Rosoff, S., 1993, «Deux-points, ouvrez les guillemets - Notes sur la ponctuation du discours rapporté au XVIIIe siècle », Le Gré des langues, 5, p. 178-202, L'Harmattan.
} 
plus en plus visuellement explicite pour le lecteur, précédant en cela les grammairiens. Néanmoins des palliatifs existent. Sonia Branca observe que ce sont souvent des interjections qui ouvrent le DD, servant ainsi de démarcatif. Pour ma part je note plutôt (ou se cumulant) la désignation du locuteur sujet, de l'allocutaire, identifié par le complément d'objet indirect ou par l'apostrophe, si bien qu'il est possible, dans les corpus observés, d'opposer de façon sommaire deux systèmes de signalement du DD, selon que le DD entre dans une construction « juxtaposée » avec le verbe de locution qui le précède (système I), ou qu'il se présente coupé ou terminé par une incise (système II $)^{14}$.

\subsection{Les deux systèmes : un cadre de départ}

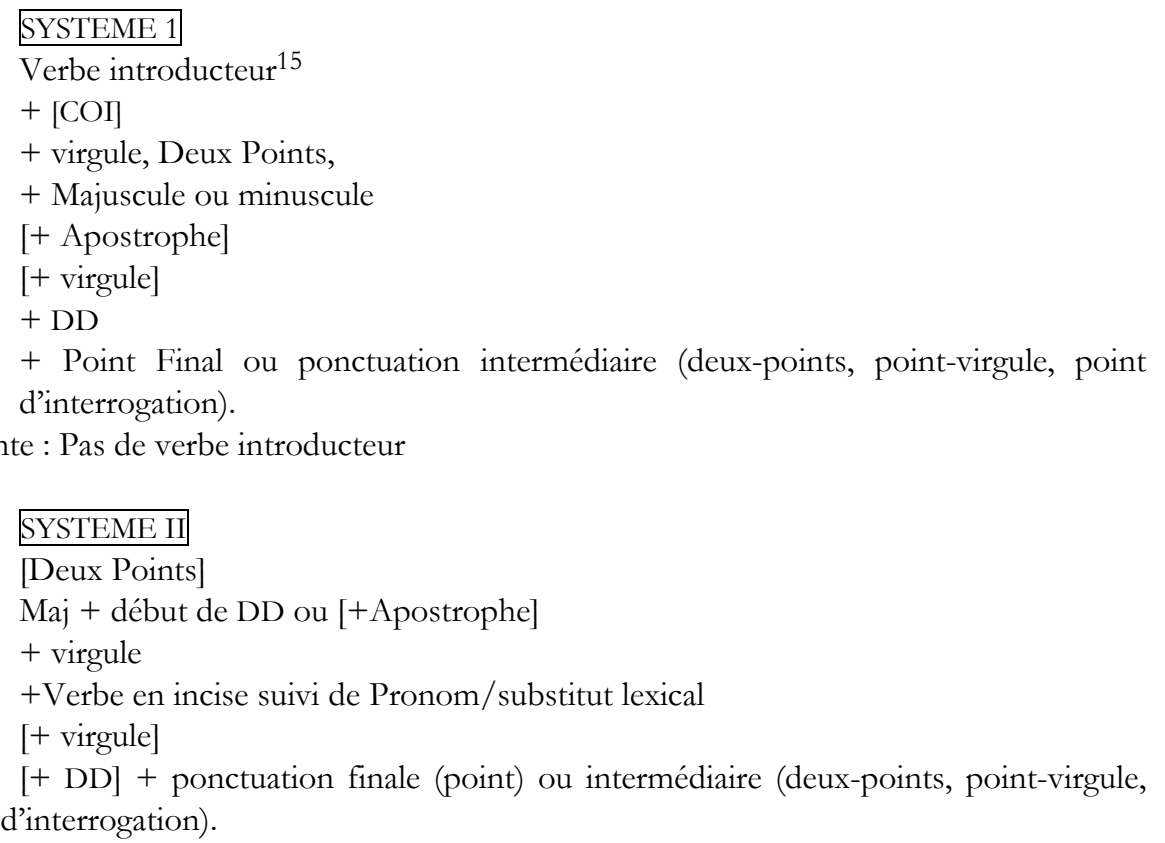
point d'interrogation).

\footnotetext{
14 Tilkin (op. cit., p. 201-202) parle d'analepses intercalées ou d'analepses de clôture pour désigner l'incise suivant sa position dans la phrase, et de prolepses pour signifier que le récit est suivi du DD. Chez Voltaire, les analepses intercalées domineraient (43\% des occurrences) alors que les prolepses ne feraient que $18 \%$ des cas. Dans le corpus Perrault, l'écart est moindre : les analepses intercalées représentent $54,5 \%$ des occurrences mais il y a $34,2 \%$ de prolepses, ce qui peut s'expliquer par la nécessité de continuer à marquer par un verbe introducteur l'annonce de paroles rapportées, en l'absence de signes démarcatifs spécifiques qui seront introduits plus tard. Mais cette hypothèse demande à être mieux étayée car le système typographique et textuel de Perrault diffère parfois de celui de ses contemporains ( $c f$. ci-après, l'usage de l'alinea, différent chez Mme d'Aulnoy et Perrault).

15 Tous les exemples analysés plus bas sont limités au verbe « dire », surreprésenté dans les corpus. Mais les deux systèmes fonctionnent aussi avec d'autres verbes de locution, en très petit nombre (moins d'une demi-douzaine), voire sans verbe introducteur (plusieurs occurrences chez Perrault).
} 
Comme on pouvait s'y attendre, avec un petit jeu de signes, les combinaisons sont nombreuses. C'est ce qu'indiquent les éléments entre crochets. Le plus intéressant est sans doute l'auxiliation des signes de ponctuation avec les constituants de phrase comme le COI et l'apostrophe, isolés par des virgules. On remarque enfin l'incertitude concernant la borne fermante dans les deux systèmes.

Ce début de description ne fait sens, cependant, que s'il s'appuie sur une réflexion concernant le DD lui-même

\subsection{Le discours direct et les grammairiens}

La grammaire de Port-Royal (1660) compare les formes du discours direct et indirect en notant l'opposition grammaticale DD $v s$ discours indirect (DI) :

Cette manière de joindre les propositions par un infinitif, ou par le quod et le que, est principalement en usage quand on rapporte les discours des autres : comme, si je veux rapporter que le roi m'a dit: je vous donnerai une charge, je ne ferai pas ordinairement ce rapport en ces termes: Le roi m'a dit, je vous donnerai une charge, en laissant les deux propositions séparées, l'une de moi, et l'autre du roi ${ }^{16}$; mais je les joindrai ensemble par un que : Le roi m'a dit qu'il me donnera une charge. Et alors, comme ce n'est plus qu'une proposition qui est de moi, je change la première personne, je donnerai, en la troisième, il donnera, et le pronom vous, qui me signifioit le roi parlant, au pronom me, qui me signifie moi parlant. [...]

Cette opposition, grammaticale telle qu'elle est décrite ci-dessus, a pourtant une origine rhétorique, comme l'a démontré Laurence Rosier $(1999,2008)$; le discours direct est employé généralement dans l'inventio et relève de la fiction, tandis que le discours indirect est typique des textes législatifs ou historiques. Le discours indirect n'est donc pas la transposition mécanique du DD à quoi il est souvent réduit :

Les calques latins oratio recta (discours direct, désormais DD) et oratio obliqua (discours indirect, désormais DI) ne formaient pas un couple grammatical soudé dans l'Antiquité. A notre connaissance aucun emploi conjoint d'oratio recta versus oratio obliqua n'est attesté chez les grammairiens de l'Antiquité.

[...] Dans l'esprit des rhéteurs, comme Quintilien, on distingue la narration d'événements historiques ou adlocutiones obliqui et la production, à des fins argumentatives et rhétoriques, de paroles fictives ou oratio recta.

Dès les origines antiques, la réflexion sur les formes de DR n'est pas spécifiquement grammaticale, elle est textuelle et discursive. (Rosier, 2008, p.11) ${ }^{17}$

Cette conception se retrouve dans la remarque finale d'Arnauld et Lancelot:

Mais il faut remarquer que les Hébreux, lors même qu'ils parlent en une autre langue, comme les Évangélistes, se servent peu de cette union des propositions, et qu'ils rapportent presque toujours les discours directement, et comme ils ont été faits; de sorte que l'« oti quod», qu'ils ne laissent pas de mettre quelquefois, ne sert souvent de rien, et ne lie point les propositions, comme il fait dans les autres auteurs. En voici un exemple dans le premier chapitre de Saint Jean: Miserunt Judai ab Hierosolymis Socerdotes et Levitas ad Joannem ut interrogarent eum: Tu quis es? Et confessus est et non

16 Je souligne.

17 On cite cependant de longs passages chez Furetière ou Bouhours avec des guillemets tout au long. 
negavit, et confessus est : quia (" oti ») non sum ego Christus, Et interrogaverunt eum : Quid ergo? Elias es tu? Et dixit: Non sum. Propheta es tu? Et respondit, non. Selon l'usage ordinaire de notre langue, on auroit rapporté indirectement ces demandes et ces réponses en cette manière. Ils envoyèrent demander à Jean qui il était. Et il confessa qu'il n'était point le Christ. Et ils lui demandèrent qui il étoit donc: s'il étoit Elie, Et il dit que non. S'il étoit Prophète, et il répondit que non ,[...]. (Chap. XVII, Grammaire générale et raisonnée, Arnauld et Lancelot, 1660)

Le discours direct est ainsi réputé animer une narration à des fins argumentatives ou rhétoriques. Dans les contes, où le DD est fictif, la voix hétérogène de l'autre est en principe dégagée de celle de l'auteur-narrateur au moyen des signes visuels de ponctuation. Mais nous savons qu'il n'existe au XVII ème siècle ni alinéa, ni tiret, ni guillemets dans le cas des discours directs de fictions.

Les recommandations de Beauzée (1765) montrent cependant qu'un ensemble de règles se dégage pour fixer une ponctuation demeurée flottante jusqu’au milieu du XVIII ${ }^{\text {ème }}$ siècle :

C'est un usage universel \& fondé en raison, de mettre les deux points après qu'on a annoncé un discours direct que l'on va rapporter, soit qu'on le cite comme ayant été dit ou écrit, soit qu'on le propose comme pouvant être dit ou par un autre ou par soi-même. Ce discours tient, comme complément, à la proposition qui l'a annoncé ; \& il y auroit une sorte d'inconséquence à l'en séparer par un point simple, qui marque une indépendance entière : mais il en est pourtant très-distingué, puisqu'il n'appartient pas à celui qui le rapporte, ou qu'il ne lui appartient qu'historiquement, au lieu que l'annonce est actuelle ; il est donc raisonnable de séparer le discours direct de l'annonce par la ponctuation la plus forte au-dessous du point, c'est-à-dire par les deux points. Exemples :

Lorsque j'entendis les scenes du paysan dans le faux généreux, je dis : "voilà qui plaira à toute la terre ¿o dans tous les tems, voilà qui fera fondre en larmes». M. Diderot, de la Poésie dramatique. [...]

Il faut remarquer que le discours direct que l'on rapporte, doit commencer par une lettre capitale, quoiqu'on ne mette pas un point à la fin de la phrase précédente. Si c'est un discours feint, comme ceux des exemples précédens, on a coûtume de le distinguer du reste par des guillemets : si c'est un discours écrit que l'on cite, il est assez ordinaire de le rapporter en un autre caractere que le reste du discours où celuilà est introduit, soit en opposant l'italique au romain, soit en opposant différens corps de caracteres, de l'une ou de l'autre de ces deux especes.

Le raisonnement grammatical de Beauzée véhicule l’idée déjà moderne que le DD comme énonciation se distingue de l'énonciation « actuelle » de son producteur, ce que la ponctuation doit, selon lui, entériner par une sorte de mise en scène visuelle (et paradoxale !) de cette voix.

Cependant, la ponctuation ancienne des Contes de Perrault et d'Aulnoy est empreinte d'une autre conception. Pour la comprendre, il faut revenir sur les systèmes I et II présentés ci-dessus.

\section{Le SYSTEME I dans les Contes}

L'introduction du DD par un verbe de locution place tout le discours cité en position rhématique, à l'inverse de l'incise, ce qui distingue fonctionnellement les deux systèmes. 
Mais la différence entre les deux systèmes vient aussi des régularités beaucoup plus grandes que l'on observe dans la borne ouvrante gauche du DD de système I.

\subsection{Borne ouvrante gauche du DD}

\subsubsection{La virgule ouvrante}

Dans le SYSTEME I, édition 1697, la borne ouvrante du DD se marque par la virgule (près de $88 \%$ des formes) :

(1) Un jour sa mere ayant cui et fait des galettes, luy dit, va voir comme se porte ta mere-grand, [...] (Le Petit Chaperon rouge, 1697)

(2) la pauvre enfant qui ne sçavoit pas qu'il est dangereux de s'arrester à écouter un Loup, luy dit, je vais voir ma Mere-grand, [...] (ibid.)

(3) Le Chat qui entendoit ce discours, mais qui n'en fit pas semblant, luy dit dun air posé et serieux, ne vous affligés point, mon maistre, [...] (Le maître Chat ou le Chat Botté, 1697)

(4) La bonne femme ayant bû, luy dit, vous estes si belle, si bonne et si honneste, [...] (Les Fées, 1697)

La minuscule est plus utilisée que la majuscule à l'initiale du premier mot du DD, ce qui accentue l'impression que le DD fait partie de la narration.

DD :

Dans l'exemple suivant, il n'y a plus aucun signe de ponctuation annonçant le

(5) Ayant presté l'oreille plus attentivement, elle ouit que l'un disoit apporte-moy cette marmite, l'autre donne-moy cette chaudiere, l'autre mets du bois dans ce feu.

(Riquet à la Houppe, 1697)

La construction directe est "intégrée » à la phrase. Le DD est illustratif (il montre trois exemples plutôt qu'il ne fait parler trois personnages) et entièrement absorbé par la structure hiérarchiquement supérieure qu'est la construction complétive après le verbe «ouïr». Le DD complément de "dire», loin d'être exhibé, est au contraire ici fondu dans l'énonciation principale du narrateur et nous allons voir que c'est là un procédé fréquent dans les Contes.

\subsubsection{Le deux points}

On trouve aussi le deux points introducteur :

(6) Dans ce moment la jeune Fée sortit de derriere la tapisserie, et dit tout haut ces paroles : Rassurez-vous Roi et Reine, [...] (La Belle au bois dormant, 1697)

mais on ne le trouvera systématiquement que dans l'édition de 1785 , où les proportions s'inversent: la virgule sera remplacée par le deux points suivi d'une majuscule dans plus de $80 \%$ des cas. L'influence des grammairiens ( $c f$. ci-dessus Beauzée) a pu se faire sentir.

Le deux point est parfois la seule façon d'annoncer un DD :

(7) Sa Maraine qui la vit toute en pleurs, luy demanda ce qu'elle avoit: Je voudrois bien... Je voudrois bien... (Cendrillon ou La Petite Pantoufle de Verre, 1697)

(8) Sa marraine, qui la vit toute en pleurs, lui demanda ce qu'elle avoit. Je voudrois bien... Je voudrois bien... (Cendrillon ou La Petite Pantoufle de Verre, 1785) 
Dans (7) et (8) on aura remarqué l'absence de verbe introducteur. De ce fait, seul le deux-points en (7) annonce le changement d'interlocuteur : il marque la rupture d'énonciation tout en soulignant l'interaction avec ce qui précède. Pour (8), c'est aux seules ruptures déictiques de signifier le $\mathrm{DD}$, qui mime parfaitement le discours autre en se substituant soudain à la voix du narrateur.

\subsection{Borne fermante du DD (système I)}

A l'extrémité droite du DD, se trouve sa borne fermante, généralement un point, du moins dans les cas simples:

(9) Cela estoit fort bien allé jusques là, mais un soir cette méchante Reine dit au Maistre-d'Hôtel, je veux manger la Reine à la mesme sausse que ses enfans. (La Belle au bois dormant, 1697)

Le point borne une réplique brève ou longue au $\mathrm{DD}$, quand celle-ci émane d'un seul personnage (cependant voir plus bas).

\subsubsection{Ponctuation de dialogue}

Mais on constate aussi que le point ne ferme le DD qu'une fois le dialogue terminé entre deux interlocuteurs :

(10) En les coëffant, elles luy disoient, Cendrillon, serois tu bien aise d'aller au Bal ? Helas, Mesdemoiselles, vous vous mocquez de moy, ce n'est pas là ce qu'il me faut: $\underline{\mathrm{tu}}$ as raison; on riroit bien, si on voyoit un Culcendron aller au Bal. (Cendrillon ou La Petite Pantoufle de Verre, 1697)

Le point final signale la fin du dialogue, compris comme texte de l'échange tout

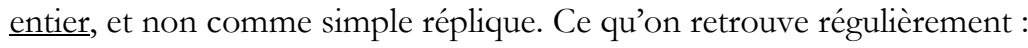

(11) La Barbe bleuë l'ayant considerée, dit à sa femme, pourquoy y a t-il du sang sur cette clef? je n'en sçais rien, répondit la pauvre femme, plus pasle que la mort: Vous n'en sçavez rien, reprit la Barbe bleuë, je le sçay bien moy, vous avez voulu entrer dans le cabinet? Hé bien, Madame, vous y entrerez, et irez prendre vostre place auprés des Dames que vous y avez veuës. (La Barbe blene, 1697)

Dans l'édition de 1785, chaque changement d'interlocuteur est séparé par un point, chaque point (y compris d'interrogation) est suivi d'une majuscule. En cas de point d'interrogation terminant une réplique, le point est exclu ${ }^{18}$ :

\footnotetext{
18 C'était déjà le cas en 1697, voir (9) ; mais ce ponctuant pouvait être suivi d'une minuscule comme d'une majuscule, ce qui montre que sa valeur délimitative n'était pas fixée.

Claude Tournier (1980, p. 39) a théorisé les contraintes de position des signes typographiques dans les lois ci-dessous. Le termes de ponctuant et ponctuance qu'il utilise infra désignent respectivement le signifiant graphique et le signifié des signes typographiques (pour une critique, voir Dahlet, 2005)

«- Loi générale : exclusion

Certains ponctuants s'excluent mutuellement, c'est-à-dire que même s'il y a, en un point du discours, plusieurs ponctuances à marquer, un seul ponctuant est réalisé, en une seule fois.

- Loi de neutralisation
} 
(12) Et les coiffant, elles lui disoient: Cendrillon, serois-tu bien-aise d'aller au bal ? Hélas, mesdemoiselles, vous vous moquez de moi: ce n'est pas-là ce qu'il me faut. $\mathrm{Tu}$ as raison; on riroit bien, si on voyoit un Cucendron aller au bal. (1785)

Mais la clarté syntaxique de 1785 qui a remplacé l'organisation textuelle de 1697 ne peut venir à bout de toutes les ambivalences. Les majuscules notent avec régularité le changement d'interlocuteur, mais elles notent aussi le début d'une phrase. La valeur (ponctuance selon C. Tournier) du point est aussi ambivalente; le point se trouve cumuler trois fonctions à interpréter : fin de phrase à l'intérieur de la réplique d'un locuteur, changement d'interlocuteur, fin de l'échange, comme on le voit dans l'exemple ci-dessous :

(13) La Barbe bleue l'ayant considérée, dit à sa femme : Pourquoi y a-t-il du sang sur cette clé ? Je n'en sais rien, répondit la pauvre femme, plus pâle que la mort. Vous n'en savez rien, reprit la Barbe bleue? je le sais bien, moi. Vous avez voulu entrer dans le cabinet? Hé bien, madame, vous y entrerez, et irez prendre votre place auprès des dames que vous y avez vues. (édition de 1785)

\subsubsection{Entre séquence et texte}

La ponctuation du discours direct est révélatrice de la conception que l'on se fait des discours rapportés au XVII ème siècle. Ainsi la ponctuation du XVII ${ }^{\text {ème }}$ siècle estelle, dans sa désinvolture apparemment anarchique pour un moderne, souvent utilisée pour sa valeur textuelle, rhétorique et dramatique.

Dans (14), par exemple, le discours direct de la reine ne trouve son achèvement qu'à la fin de l'épisode de la tromperie du maitre d'hôtel : il clôture une séquence, scandée par le deux-points, ou le point-virgule, de force moindre que le point.

(14) Huit jours aprés la méchante Reine dit à son Maistre-d'Hôtel, je veux manger à mon souper le petit Jour: il ne repliqua pas, résolu de la tromper comme l'autre fois ; il alla chercher le petit Jour, et le trouva avec un petit fleuret à la main, dont il faisoit des armes avec un gros Singe, il n'avoit pourtant que trois ans : il le porta à sa femme qui le cacha avec la petite Aurore, et donna à la place du petit Jour, un petit chevreau fort tendre, que l'Ogresse trouva admirablement bon. (1697)

Plusieurs exemples le montrent, plus ou moins développés selon la séquence :

(15) Cendrillon qui les regardoit, et qui reconnut sa pantoufle, dit en riant, que je voye si elle ne me seroit pas bonne : ses soeurs se mirent à rire et à se moquer d'elle. (Cendrillon ou La Petite Pantoufle de Verre, 1697)

(16) Ensuite elle luy dit, va dans le jardin, tu y trouveras six lezards derriere l'arrosoir, apporte-les-moy, elle ne les eut pas plûtost apportez, que la Maraine les changea en

\footnotetext{
Si en un point du discours plusieurs ponctuances doivent être marquées, et ne peuvent normalement l'être que par le même ponctuant, celui-ci n'est réalisé qu'une fois.

- Loi d'absorption

Il existe des signes qui ne peuvent apparaître que l'un à côté de l'autre bien que comportant des ponctuants et des ponctuances différents : dans de tels cas, un seul ponctuant est réalisé et il se charge alors de sa ponctuance propre et des autres ».
} 
six Laquais qui monterent aussi-tost derriere le carosse avec leurs habits chamarez, et qui s'y tenoient attachez, comme s'ils n'eussent fait autre chose toute leur vie. (ibidem)

Ce qui donne dans l'édition de 1785 :

(17) Cendrillon, qui les regardoit, et qui reconnut sa pantoufle, dit en riant: Que je voie si elle ne me seroit pas bonne. Ses sœurs se mirent à rire et à se moquer d'elle. (1785)

(18) Ensuite elle lui dit: Va dans le jardin, tu y trouveras six lézards derrière l'arrosoir, apporte-les moi. Elle ne les eut pas plutôt apportés, que la marraine les changea en six laquais, qui montèrent aussitôt derrière le carrosse avec leurs habits chamarrés, et qui s'y tenoient attachés, comme s'ils n'eussent fait autre chose toute leur vie. (1785)

L'édition du Cabinet des Fées segmente en phrases grammaticales, là où l'édition de 1697 montrait, par des pauses de force moindre, l'unité de sens de la séquence englobante. Mais cela ne se vérifie pas partout: la séquence peut être interrompue pour des raisons de dramatisation. En (19) nous sommes dans la variante du système 1 où le verbe d'introduction a été mis en «facteur commun » :

(19) Le petit chaperon rouge se deshabille, et va se mettre dans le lit, où elle fut bien estonnée de voir comment sa Mere-grand estoit faite en son deshabillé, elle luy dit, ma mere-grand que vous avez de grands bras! c'est pour mieux t'embrasser, ma fille: ma mere-grand que vous avez de grandes jambes? c'est pour mieux courir mon enfant: ma mere-grand que vous avez de grandes oreilles? c'est pour mieux écouter mon enfant. (Le Petit Chaperon rouge, 1697)

Restent deux phrases de dialogue isolées par le point:

(20) Ma mere-grand que vous avez de grands yeux ? c'est pour mieux voir, mon enfant. Ma mere-grand que vous avez de grandes dens ? c'est pour te manger. (1697)

Cette ponctuation n'est pas fantaisiste, il semble qu'elle obéisse à une intention d'auteur. En effet, le manuscrit de 1695 ( $c f$. Annexe) arrête l'épisode par un point au même endroit que (19). On voit aussi dans le manuscrit la célèbre adjonction en marge spécifiant la manière de prononcer la dernière phrase destinée à effrayer l'enfant. Ces indices laissent penser que si (20) diffère sémantiquement et rhétoriquement de (19), la ponctuation a pu le marquer par une pause qui sert la dramatisation.

Par ailleurs, l'hésitation entre le point d'interrogation et le point admiratif (d'exclamation) que l'on trouve dans ce passage est réglée par l'uniformité du point d'exclamation dans l'édition de 1785 de Cabinet des Fées (21). Ce simple fait résout peutêtre une inadvertance mais marque surtout la nouvelle valeur distinctive de ce signe, encore employé de façon indifférenciée au XVII ème siècle en alternance avec le point interrogant.

(21) Le petit Chaperon rouge se déshabille, et va se mettre dans le lit, où elle fut bien étonnée de voir comment sa mère grand' étoit faite en son déshabillé. Elle lui dit: Ma mère-grand', que vous avez de grands bras! - C'est pour mieux t'embrasser, ma fille. - Ma mère grand', que vous avez de grandes jambes! - C'est pour mieux courir, mon enfant. - Ma mère grand', que vous avez de grandes oreilles! - C'est pour mieux écouter, mon enfant. - Ma mère grand', que vous avez de grands yeux ! 
— C'est pour mieux voir, mon enfant. — Ma mère grand', que vous avez de grandes dents! - C'est pour te manger.

On remarque particulièrement la rationalisation : le texte est désormais découpé et équipé de tirets marquant l'alternance des voix. En revanche, aucune hiérarchie ne permet de distinguer pauses et nuances dans le dialogue représenté. Ou bien faut-il conclure que désormais l'initiative est aux personnages - et non plus à l'auteur -, personnages théâtralement et dialogiquement montrés dans la version graphique de leur voix?

\section{Le SYSTEME II dans les Contes}

Ce que j'ai appelé SYSTÈME II note les incises. Elles sont très abondantes dans les deux corpus Perrault-Aulnoy, et cela n'est pas sans raison: on trouvera chez Marmontel ${ }^{19}$ une explication de la présence jugée par lui envahissante des incises de discours direct :

Il n'est aucun genre de narration où le discours direct ne soit en usage, \& il y répand une grace \& une force qui n'appartient qu'à lui. Mais dans le dialogue pressé, il a un inconvénient auquel il seroit aussi avantageux que facile de remédier. C'est la répétition fatigante de ces façons de parler, lui dis-je, reprit-il, me répondit-elle, interruptions qui rallentissent la vivacité du dialogue, \& rendent le style languissant où il devroit être le plus animé. Quelques anciens, comme Horace, se sont contentés dans la narration, de ponctuer le dialogue. Mais ce n'étoit point assez pour éviter la confusion. Quelques modernes, comme la Fontaine, ont distingué les répliques par les noms des interlocuteurs; mais cet usage ne s'est introduit que dans les récits en vers. Le moyen le plus court \& le plus sûr d'éviter en même tems les longueurs \& l'équivoque, seroit de convenir d'un caractere qui marqueroit le changement d'interlocuteurs, \& qui ne seroit jamais employé qu'à cet usage.

Les caractères suggérés seront proposés par Beauzée dans l'article déjà cité, à savoir tirets et guillemets.

Cependant, si l'on observe «l'arsenal» déployé par nos auteurs pour signaler le DD grâce à l'incise, on trouve un système cohérent. En fait, d'autres éléments accompagnent la ponctuation pour permettre au lecteur de comprendre le texte avant la généralisation ultérieure des tirets et des guillemets. L'ordre des mots, beaucoup plus souple que dans le système I, insère le DD et pointe sur l'allocutaire par l'« apostrophe » en des points insolites du texte, déportant la clôture du discours très au-delà des limites de la phrase citée.

En voici un exemple en (22) :

(22) Un jour qu'elle estoit à cette fontaine, il vint à elle une pauvre femme qui la pria de lüy donner à boire? Ouy da, ma bonne mere, dit cette belle fille, et rinçant aussi tost sa cruche, elle puisa de l'eau au plus bel endroit de la fontaine, et la lui presenta, soûtenant toûjours la cruche afin qu'elle bût plus aisément. (Les Fées, 1697)

19 Article « Direct » de l'Encyclopédie (t. 4, 1754). 


\section{Dans l'édition de 1785 :}

(23) Un jour qu'elle étoit à cette fontaine, il vint à elle une pauvre femme qui la pria de lui donner à boire. Oui-dà, ma bonne mère, dit cette belle fille; et rinçant aussitôt sa cruche, elle puisa de l'eau au plus bel endroit de la fontaine, et la lui présenta, soutenant toujours la cruche, afin qu'elle bût plus aisément. (Les Fées, 1785)

L'incise est souvent entre virgules mais celles-ci n'ont pas toutes le même statut; tout dépend de la place de l'incise. A cet égard, (22) est caractéristique des ambiguités de lecture. L'incise entre virgules « coupe » la phrase de DD qu'elle signale. Mais quant l'incise est postposée au DD et en position finale comme dans (22), on peut en faire plusieurs lectures. Elle peut coïncider avec la fin de la proposition : c'est ainsi que l'interprète (23), qui semble considérer la phrase commencée par le DD comme une période dont le premier membre s'arrête après l'incise. La ponctuation de (22) me paraît plus indécise : d'abord du fait du point d'interrogation ${ }^{20}$ qui absorbe toute autre ponctuation, et initie une longue séquence. Dès lors, la virgule située à droite de l'incise cumule deux fonctions ; tout d'abord, le marquage de l'incise et du nom de locuteur, ce qui rétroactivement signale aussi le DD, en l'absence de tiret et de guillemets ; cette virgule marque aussi une pause (respiratoire ?) dans la période, qui enchaîne les deux prédicats successifs de «dire» puis de "puiser de l'eau », etc. L'édition de 1785 ne fait que marquer grammaticalement cette partition.

En résumé, quelles sont les caractéristiques, distinctives de celles de 1697, de l'édition du Cabinet des Fées (1785) chez Perrault? Je les ramènerais aux points suivants :

- généralisation du deux points + majuscule à la borne gauche du système I.

C'est encore plus vrai pour le système II, l'insertion du DD étant désormais séparée du reste de la phrase par une ponctuation forte (le point, au lieu du deuxpoints ou du point-virgule):

A ce titre on peut comparer l'édition de 1697 :

(24) La chose réüssit comme il l'avoit pensé; car l'Ogre s'étant éveillé sur le minuit, eut regret d'avoir differé au lendemain ce qu'il pouvoit executer la veille, il se jetta donc brusquement hors du lit, et prenant son grand Couteau, allons-voir, dit il, comment se portent nos petits drolles, n'en faisons pas à deux fois; il monta donc à tâtons à la Chambre de ses filles et s'approcha du lit où étoient les petits garçons, qui dormoient tous excepté le petit Pouçet, qui eut bien peur lors qu'il sentit la main de l'Ogre qui luy tastoit la teste, comme il avoit tasté celle de tous ses freres. (Le Petit Poucet, 1697)

à celle de 1785 :

\footnotetext{
${ }^{20}$ Le point d'interrogation note l'interrogation directe ou indirecte (comme ici), ce qui sera proscrit par Beauzée dans son article «Ponctuation» de l'Encyclopédie : «Si la phrase interrogative n'est pas directe, \& que la forme en soit rendue dépendante de la constitution grammaticale de la proposition expositive où elle est rapportée ; on ne doit pas mettre le point interrogatif : la ponctuation appartient à la proposition principale, dans laquelle celle-ci n'est qu'incidente. Mentor demanda ensuite à Idomenée quelle étoit la conduite de Protesilas dans ce changement des affaires. Télémaque, l. XIII.»
} 
(25) Il se jeta donc brusquement hors du lit, et prenant son grand couteau : Allons voir, dit-il, comment se portent nos petits drôles; n'en faisons pas à deux fois. (Le Petit Poucet, 1785)

- rationalisation du dialogue par utilisation du tiret quand c'est possible, sinon du point.

- le point coupe le dialogue en assignant à chaque locuteur sa phrase. Dans le cas d'une réplique longue, le point-virgule hiérarchise les éléments inclus dans la période.

- le point d'interrogation marque systématiquement les questions directes, de moins en moins les indirectes.

- utilisation systématique de l'italique pour les formules répétitives en DD: Bonnes gens qui moissonnez, Anne ma sæur Anne, etc, qui apparaissent ainsi clairement autonymiques.

- la capitalisation mériterait un long développement ${ }^{21}$. Son caractère distinctif dans le DD (une majuscule est mise à l'apostrophe presque systématiquement en 1697) se confond quelquefois avec la position initiale, dans la borne ouvrante de gauche du $\mathrm{DD}$, ou même avec la première lettre du prénom désignant l'allocutaire : la valeur de la majuscule comme ouverture du DD, en système I ou II, ne sera fixée que dans la seconde moitié du XVIII ème siècle.

En bref, dans la version imprimée de 1785, dominent hiérarchisation et fragmentation du discours par une ponctuation devenue rationnelle et figurative: celle-ci souligne les rapports hiérarchiques entre les parties, laissant des traces pour le lecteur, tandis qu'au contraire l'édition de 1697 est le fait du locuteur premier, enrobant dans son discours celui de l'autre et l'absorbant.

Quelques incursions dans le corpus Aulnoy permettent de confirmer une évolution comparable pour le DD, entre le tout début du XVIII ${ }^{\mathrm{ème}}$ siècle et l'édition de 1785. On notera cependant une variante d'auteur extrêmement spécifique : l'alinéa.

\section{Un « coup de sonde »: Mme d'Aulnoy et la ponctuation de DD 5.1. «Finette Cendron» dans les deux systèmes}

Pour Mme d'Aulnoy, je m'en suis tenue au seul conte de «Finette Cendron» pour lequel je disposais des trois éditions précitées $(1710,1757,1785)$.

Or, l'on constate que les principes de l'édition Barbin, valables pour Perrault, sont encore conservés dans l'édition de la Compagnie des Libraires sur le corpus

\footnotetext{
${ }^{21}$ Je laisse de côté le problème des majuscules hors DD pléthoriques dans les textes du XVIİ̀me siècle. On notera une approche descriptive diachronique de A. Husson (1977) sur les choix typographiques des imprimeurs en rapport (ou non) avec les recommandations des grammairiens.

Une étude structurale de D. Gilson (1981) montre que le conte de Perrault les Fées est construit sur une opposition entre les personnages et lieux bénéfiques pourvus d'une majuscule, et les négatifs écrits en minuscule, ce que retrace leur parcours narratif.

La seule approche linguistique est celle de A. Eskénazi (2000) qui porte sur les majuscules dans Les fourberies de Scapin.
} 
Aulnoy, comme en témoigne la comparaison sur un très court extrait concernant les deux systèmes et notamment la borne droite du DD.

Edition de 1710 (Barbin, veuve Ricœur, Paris)

(26) Finette dit au cheval : Mon petit ami vous êtes beau \& très sage, vous allez plus vite que le Soleil, je vous remercie de vôtre peine, retournez d'où vous venez; elle entra tout doucement dans la maison, cachant son sac sous son chevet, elle se coucha sans faire semblant de rien; dès que le jour parut, le Roi réveilla sa femme : Allons, allons, Madame, lui dit-il, apprêtez-vous pour le voïage ; aussi-tôt elle se leva, prit ses gros souliers, une juppe courte, une camisolle blanche \& un bâton; elle fit venir l'aînée de ses filles, qui s'appeloit Fleur-d'amour ; la seconde Belle-de-Nuit \& la troisième Fine-Oreille, c'est pourquoi on la nommait ordinairement Finette.

Édition de 1757 (Compagnie des Libraires, Paris)

(27) Finette dit au cheval : mon petit ami, vous êtes beau \& très sage, vous allez plus vite que le Soleil, je vous remercie de votre peine, retournez d'où vous venez: elle entra tout doucement dans la maison, cachant son sac sous son chevet: elle se coucha sans faire semblant de rien; dès que le jour parut, le Roi réveilla sa femme : Allons, allons, Madame, lui dit-il, apprêtez-vous pour le voyage; aussi-tôt elle se leva, prit ses gros souliers, une juppe courte, une camisole blanche \& un bâton; elle fit venir l'ainnée de ses filles qui s'appelait Fleur-d'amour; la seconde Belle-de-Nuit et la troisième Fine-Oreille; $c^{\prime}$ 'est pourquoi on la nommoit ordinairement Finette.

Comme on le voit, au milieu du XVIII ème siècle, le DD de système I est encore largement intégré dans le tissu textuel mais trente ans plus tard, les principes clarificateurs et hiérarchiques des grammairiens ont fait école chez les imprimeurs :

Édition de 1785 (Cabinet des Fées, Amsterdam) :

(28) Finette dit au cheval: Mon petit ami, vous êtes beau et très-sage; vous allez plus vite que le soleil ; je vous remercie de votre peine; retournez d'où vous venez. Elle entra tout doucement dans la maison, cachant son sac sous son chevet; elle se coucha sans faire semblant de rien. Dès que le jour parut, le roi réveilla sa femme: Allons, allons, madame, lui dit-il, apprêtez-vous pour le voyage. Aussitôt elle se leva, prit ses gros souliers, une juppe courte, une camisole blanche et un bâton. Elle fit venir l'aînée de ses filles qui s'appeloit Fleur-d'Amour, la seconde Belle-de-Nuit et la troisième Fine-Oreille : c'est pourquoi on la nommoit ordinairement Finette.

L'évolution des systèmes conduit aux mêmes conclusions : le discours représenté est interprété et relève d'une mise en scène soulignée par le narrateur: l'illusion référentielle donne à lire des discours soutenus par une ponctuation qui les rationalise.

\subsection{L'alinéa chez Mme d'Aulnoy}

Cependant, une caractéristique d'auteur se dégage du corpus d'Aulnoy avec la dominance d'un signe ultime de ponctuation, le blanc avec retrait qu'est l'alinéa +paragraphe narratif ${ }^{22}$.

\footnotetext{
22 Longuement étudié par M. Arabyan (1994) : Perrault constitue, semble-t-il, une exception dans ce choix de compacité du texte.
} 
Une constatation s'impose en effet : Perrault sous-ponctue là où Mme d'Aulnoy sur-ponctue. Chez Perrault par exemple, le texte est compact et la division en paragraphes n'excède jamais 5 pour son conte le plus long Le Petit Poucet (3604 mots), tandis que toutes les plus anciennes éditions des Contes de Mme d'Aulnoy en donnent 35 pour La Bonne Petite Souris un de ses contes les plus courts (5041mots). Fait important: on ne note aucune différence dans le nombre des paragraphes entre les deux éditions de référence des deux auteurs.

On doit donc en conclure que ce trait oppositif correspond à une organisation différente du matériau narratif chez les deux conteurs, et dénote sans doute un trait stylistique et/ou sous-générique distinguant deux types de contes ou de conteurs.

Existe-t-il un rapport entre le DD et la division en paragraphes? Chez Mme d'Aulnoy comme chez Perrault les dialogues sont tout aussi fréquents; mais chez Perrault, leur traitement ne donne jamais lieu à un décrochage textuel, les DD étant profondément inscrits dans le tissu textuel.

En revanche, indication intéressante d'un point de vue textuel, il semble que le paragraphe chez Mme d'Aulnoy ait une fonction narrative incluant les événements de parole qui lui sont subordonnés, mais sans les détacher encore comme le font nos modernes éditions par l'alinéa, le tiret et les guillemets. Le paragraphe correspondrait chez cet auteur à ce que M. Arabyan (1994, p. 259), reprenant Bakhtine, nomme « la trace d'un dialogue sous-jacent entre l'auteur et son lecteur»; le lecteur, en tiers témoin du dialogue entre les personnages, est aussi pris par la main à chaque nouvel épisode.

\section{Conclusion}

Que nous apprennent les changements diachroniques de ponctuation du DD survenus dans un genre comme le conte, qui a dû être lu et interprété par ses lecteurs aussi bien à l'écrit qu'à l'oral ?

Il semble que l'évolution dans le marquage du discours de l'autre, telle que nous l'avons vue, pose plusieurs questions impliquant le dialogisme. Le dialogisme est en effet nécessairement attaché au DD, comme segment étranger au discours du locuteur; mais il n'est pas évident que ce dernier ait voulu ou pu le marquer comme tel: les textes des Contes sont en effet constamment l'objet de l'interprétation d'un lecteur, dont le premier est l'imprimeur.

L'hypothèse pragmatique exprime une conception de la ponctuation comme signal, que ce soit à l'oral (où la ponctuation indiquerait les pauses pour respirer) ou comme marque typovisuelle : la ponctuation marquerait clairement l'emplacement des DR étrangers à la parole du narrateur.

Ces explications paraissent évidentes et l'évolution diachronique marquerait alors un glissement de la lecture à haute voix vers une lecture visuelle réorganisant 
l'espace sémiotique ${ }^{23}$. Mais il me semble que la ponctuation de DD dans les textes anciens engage aussi et peut-être surtout la question de la mimesis.

Comment faut-il nommer, en effet, le responsable de la ponctuation de ces discours fictifs : auteur ou narrateur ${ }^{24}$ ? Le premier organise le matériau narratif avant tout, en y soumettant les paroles de ses personnages. Il groupe et dégroupe les propositions et les phrases à son gré pour (faire) signifier séquences et épisodes. Le second crée l'illusion de la parole en mimant du langage, ou du moins l'expressivité qui seule est «mimable » : point d'interrogation, d'exclamation, de suspension sont le fait du personnage qui s'échappe du discours auctorial pour être représenté.

Les deux éditions comparées me semblent montrer ce basculement dans la représentation des discours du personnage. La ponctuation du DD change leur statut. Selon que l'on gomme ou souligne leur autonomie, on en fait la propriété de l'auteur, ou celle d'un narrateur qui en exhibant leur hétérogénéité souligne l'illusion référentielle.

\footnotetext{
${ }^{23}$ Hypothèse défendue entre autres par Branca-Rosoff (1993, op. cit.) qui ajoute l'intéressante précision que le discours direct désormais mis en relief par la démarcation typographique, attribue au locuteur une responsabilité quasi « juridique » de sa parole.

${ }^{24}$ Ce n'est qu'un des noms de couples possibles : locuteur / énonciateur marque encore autrement la division inépuisable de l’instance énonçante.
} 
Remarques sur la ponctuation du discours direct dans les Contes de Perrault et de Mme d'Aulnoy

\section{RÉFÉRENCES BIBLIOGRAPHIQUES}

\section{Editions des contes}

Aulnoy, Marie-Catherine Le Jumel de Barneville, comtesse d', 1785, 1ère éd. 1697, Le Cabinet des fées Amsterdam, Paris, Classiques Garnier Numérique.

Barchilon, J., 1956, Perrault's Tales of Mother Goose, The dedicated manuscript of 1695 reproduced in collotype facsimile with introduction to critical text, New York, The Pierpont Morgan Library (2 vol.)

Perrault, Ch., 1697, Histoires ou contes du temps passé. Avec des Moralitę, Paris, Claude Barbin, Classiques Garnier Numérique.

ANIS, J., 1988, L'écriture, théorie et descriptions, Bruxelles, De Boeck-Wesmael.

- 2000, "Vers une sémiolinguistique de l'écrit» Linx $\mathrm{n}^{\circ} 43$ (Linguistique de l'écrit, linguistique du texte), Anis. J. \& Jeandillou, J.-F. (eds), p. 29-45.

ARABYAN, M., 1994, Le paragraphe narratif, Paris, L'Harmattan.

AUTHIER-REVUZ, J., 2004, « Le fait autonymique : langage, langue, discours- quelques repères », in Parler des mots, le fait autonymique en discours, Presses Universitaires de la Sorbonne nouvelle, p. 67-96.

BARKO, I., 1977, «Contribution à l'étude de la ponctuation française au XVII ${ }^{\text {ème }}$ siècle, problèmes de méthode, la ponctuation de Racine ", in Catach, N. \& Tournier, Cl. (dir.), La Ponctuation, recherches historiques et actuelles, Paris-Besançon, CNRS-HESO, p. 59-126.

CATACH, N., 1994, La Ponctuation, Paris, PUF, «Que sais-je ? »

Dahlet, V., 2003, Ponctuation et énonciation, Ibis Rouge éd., Presses Universitaires créoles.

Defays, J.-M., Rosier L., Tilkin, F, (eds), 1998, A qui appartient la ponctuation? Bruxelles, De Boeck et Larcier, coll. « Champs linguistiques ».

Durrer, S., 1990, «Le dialogue romanesque : essai de typologie », Pratiques n 65, « Paroles de personnages », p. 37-62.

ESKÉNAZI, A., 2000, «Les majuscules dans les Fourberies de Scapin (édition de 1674) », Linx $\mathrm{n}^{\circ} 43$ (Linguistique de l'écrit, linguistique du texte), Anis. J. \& Jeandillou, J.-F. (eds), p. 86-102.

GiLSON, D., 1981, «Le jeu des adjectifs et des majuscules » dans Les Fées de Charles Perrault », in G. Jacques (dir.), Recherches sur le conte merveilleux, Louvain-La-Neuve, p. 57-71.

Husson, A., 1977, «Une approche graphique de la majuscule et de son emploi », in Catach, N. \& Tournier, Cl. (dir.), La Ponctuation, recherches historiques et actuelles, Paris-Besançon, CNRSHESO, p. 151-167.

Rosier, L., 2008, Le discours rapporté en français, Paris, Ophrys, coll. « L'essentiel ».

- 1999, Le discours rapporté, histoires, théories, pratiques, Bruxelles, Duculot, coll. "Champs linguistiques ».

TILKIN , F., 1998, « Ponctuation et récit de paroles dans les éditions originales des Contes de Voltaire », in Defays et al. (eds.), p. 199-210.

TOURnIER, Cl., 1980, «Histoire des idées sur la ponctuation, des débuts de l'imprimerie à nos jours », Langue Française n ${ }^{\circ} 45$, p. 28-40. 
ANNEXE - Le manuscrit de Perrault 1695
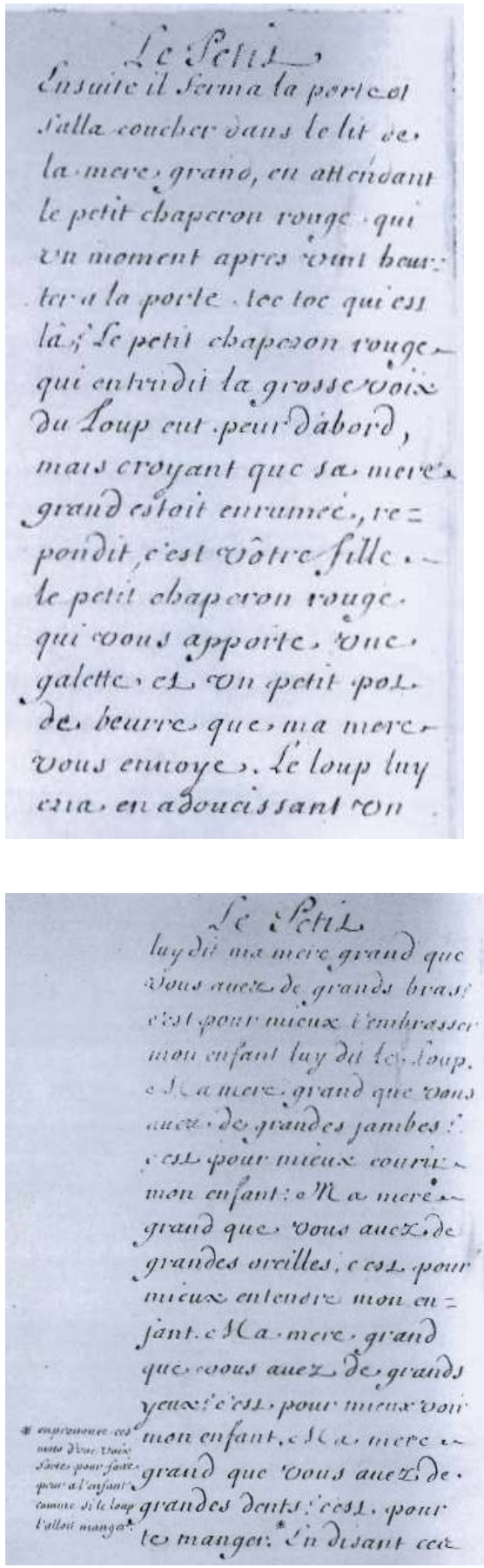

Clateron ronge: pret is verix, hice la bubi=

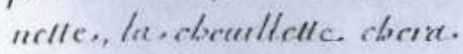
lo perit cloaperon iverges tira. ta clocuilletles is la portes de la maxison

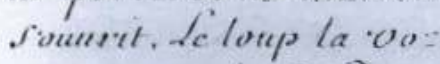
yaut entrar luy dot de. Jedaus le lis ou il des car : dooi sous la comeriture mess la galctte es le ipe: hit qoi de beurre, sur la. buche a roiens te coucher.

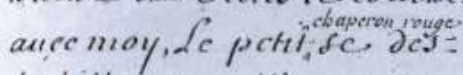
babillcies. silla. meltie. Jedans le lis, ou elle fur been ctonnce De you commens. sa. mere grand esfort faile en son desbabille; ílen

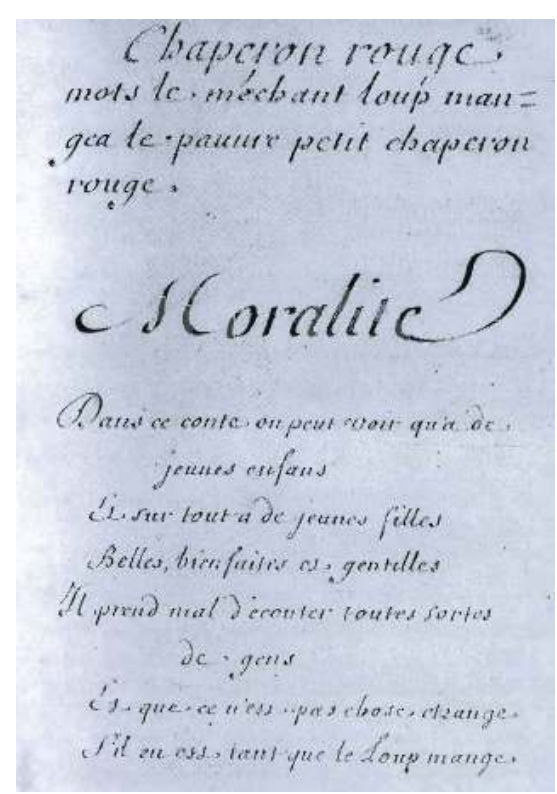

